\title{
The Effects of Anthropogenic Sound and Artificial Light Exposure on Microbiomes: Ecological and Public Health Implications
}

\author{
Jake M. Robinson ${ }^{1,2,3 *}$, Ross Cameron ${ }^{1}$ and Brenda Parker ${ }^{4,5}$ \\ ${ }^{1}$ Department of Landscape Architecture, The University of Sheffield, Sheffield, United Kingdom, ${ }^{2}$ In vivo Planetary Health, \\ Worldwide Universities Network (WUN), West New York, NJ, United States, ${ }^{3}$ The Healthy Urban Microbiome Initiative \\ (HUMI), Adelaide, SA, Australia, ${ }^{4}$ Bio-Integrated Design Lab, Bartlett School of Architecture, Here East, Queen Elizabeth \\ Olympic Park, London, London, United Kingdom, ${ }^{5}$ Department of Biochemical Engineering, University College London, \\ United Kingdom
}

\section{OPEN ACCESS}

Edited by:

Zhenhong Wang,

Guizhou University, China

Reviewed by:

Mohammed Rashed, National Research Centre, Egypt

Dan Xing,

Independent Researcher, Guizhou,

China

${ }^{*}$ Correspondence:

Jake M. Robinson

jmrobinson3@sheffield.ac.uk

Specialty section:

This article was submitted to

Conservation and Restoration

Ecology,

a section of the journa

Frontiers in Ecology and Evolution

Received: 01 February 2021

Accepted: 30 April 2021

Published: 24 May 2021

Citation:

Robinson JM, Cameron R and

Parker B (2021) The Effects

of Anthropogenic Sound and Artificial

Light Exposure on Microbiomes:

Ecological and Public Health

Implications.

Front. Ecol. Evol. 9:662588.

doi: 10.3389/fevo.2021.662588
Globally, anthropogenic sound and artificial light pollution have increased to alarming levels. Evidence suggests that these can disrupt critical processes that impact ecosystems and human health. However, limited focus has been given to the potential effects of sound and artificial light pollution on microbiomes. Microbial communities are the foundations of our ecosystems. They are essential for human health and provide myriad ecosystem services. Therefore, disruption to microbiomes by anthropogenic sound and artificial light could have important ecological and human health implications. In this mini-review, we provide a critical appraisal of available scientific literature on the effects of anthropogenic sound and light exposure on microorganisms and discuss the potential ecological and human health implications. Our mini-review shows that a limited number of studies have been carried out to investigate the effects of anthropogenic sound and light pollution on microbiomes. However, based on these studies, it is evident that anthropogenic sound and light pollution have the potential to significantly influence ecosystems and human health via microbial interactions. Many of the studies suffered from modest sample sizes, suboptimal experiments designs, and some of the bioinformatics approaches used are now outdated. These factors should be improved in future studies. This is an emerging and severely underexplored area of research that could have important implications for global ecosystems and public health. Finally, we also propose the photo-sonic restoration hypothesis: does restoring natural levels of light and sound help to restore microbiomes and ecosystem stability?

Keywords: sound pollution, artificial light, light pollution, restoration, microbiome

\section{INTRODUCTION}

Globally, anthropogenic sound pollution (e.g., from traffic and construction) has increased to alarming levels (Tabraiz et al., 2015; Kumar et al., 2018; Vitkauskaite and Grubliauskas, 2018). Moreover, the rise in artificial light pollution-such as excessive light from buildings and streets, and lighting associated with industry and transportation-is now considered to be a global health concern (Falchi et al., 2019). In terms of direct human health implications, ALAN and sound pollution have been linked to depression (Min and Min, 2018; Díaz et al., 2020) and insomnia by disrupting circadian rhythms (Hatori et al., 2017; Janson et al., 2020). Research also suggests 
that sound pollution is a biological stressor that can induce cardiovascular and endocrinological disorders (Münzel et al., 2018). For context, hazardous noise to humans is considered to be $>85$ decibels $(\mathrm{dB})$, and lawn mowers or motorcycles emit $\sim 90 \mathrm{~dB}$ (Chepesiuk, 2005).

Anthropogenic sound and artificial light pollution also have a range of impacts on ecosystem processes. For example, it is well-documented that anthropogenic sound exposure affects wildlife populations. Indeed, noise-induced reductions in foraging efficiency have been demonstrated in bats (Luo et al., 2015), owls (Mason et al., 2016), flounder larvae Pseudopleuronectes americanus (Gendron et al., 2020), and crabs (Wale et al., 2013). Chronic traffic noise can alter gene expression in bats, which associates with metabolic dysregulation and stress (Song et al., 2020). Artificial light at night (ALAN) can affect insect movement, foraging, reproduction and predatory behavior (Owens et al., 2020) and may represent broader disturbances to ecosystems by disrupting mutualistic interactions across trophic levels (Maggi et al., 2020b).

However, limited attention has been given to the potential effects of sound pollution and ALAN on microbiomes. Microbial communities are the foundations of our ecosystems and provide essential ecosystem services. These include carbon and nutrient cycling, climate regulation, global food security, and animal and plant health (Cavicchioli et al., 2019; Guerra et al., 2020; Li et al., 2020; Trivedi et al., 2020). Anthropogenic ecosystem degradation disturbs many organisms, therefore, understanding how environmental microbiomes may be affected by anthropogenic sound and artificial light could have important ecological and human health implications. The human microbiome (the network of microbial communities in the human body) is essential for human health (Sharma and Im, 2020). Indeed, a dysfunctional microbiome has been linked to a plethora of diseases from Alzheimer's (Kowalski and Mulak, 2019), skin diseases (Prescott et al., 2017) and mental health conditions (Cryan et al., 2019). Furthermore, exposure to diverse environmental microbiomes is thought to play a role in "training" and regulating the immune system (Rook, 2013; Renz and Skevaki, 2020; Robinson and Jorgensen, 2020; Roslund et al., 2020).

Therefore, disturbance to environmental and human microbiomes by anthropogenic sound and artificial light pollution, could have important implications for both ecosystem functionality and human health. In this mini-review, we provide a critical appraisal of available scientific literature on the effects of anthropogenic sound and artificial light exposure on microorganisms. We discuss the potential ecological and human health implications of these effects.

\section{THE EFFECTS OF ANTHROPOGENIC SOUND EXPOSURE ON MICROORGANISMS}

\section{Bacteria}

We found 12 scientific papers pertaining to the exposure of anthropogenic sound on bacteria, 8 of which will be discussed and the other 4 were excluded due to a lack of data and relevance. A pilot study by Shaobin et al. (2010) investigated the effects of audible sound on Escherichia coli growth. The authors placed cultured $E$. coli cells ( $n=15$ plates) into sound chambers and stimulated them using $90 \mathrm{~dB}$ sound waves (similar levels to urban traffic). They applied frequencies of 1,5 , and $15 \mathrm{kHz}$ for $1 \mathrm{~h}$ periods with $3 \mathrm{~h}$ intervals over a $24 \mathrm{~h}$ treatment period. They found that E. coli growth was significantly promoted and reached colony forming efficiencies of 142, 130, and $131 \%$ after sound stimulation with 1,5 , and $15 \mathrm{kHz}$, respectively. Although the sample size was modest, this study was later corroborated by $\mathrm{Gu}$ et al. (2016) who found that E. coli K-12 ( $n=100$ randomly selected cells) exposed to $80 \mathrm{~dB}$ sound waves exhibited increased biomass and growth rate at $8 \mathrm{kHz}$ by $1.7 \times$ and $2.5 \times$ (compared to the control), respectively. While variations in the inoculum could impact growth rates, further studies making use of high throughput cell cultivation strategies could be employed to improve robustness. However, the mechanism of sound stimulation on microbial growth is still unknown-therefore further research is required. Similar experiments could conceivably be carried out to investigate microbiome compositional changes and explore different interfaces and media that may affect sound propensity (e.g., water and soil) (Figure 1). Mechanosensitive channels on bacterial cell membranes might be involved in signal transduction which provides a promising area to focus on. Interestingly, Kim (2016) found that antibiotic resistance to ampicillin increased in soil bacteria $(n=10)$ and E. coli $(n=10)$ exposed to low frequency sound $(75 \mathrm{~dB}$ at $0.1 \mathrm{kHz})$. The sample size in this study is modest, therefore, caution is needed. However, the indication of increased antibiotic resistance attributed to low frequency anthropogenic noise, warrants further research. The authors conclude that the amount of soil bacteria exposed to noise also increased but chlorophyll optical density (of associated plants) was unaffected. Therefore, it is possible that soil bacteria with mutualistic plant interactions such as nitrogen fixation and denitrification, were outcompeted by less useful bacteria. This also warrants more research due to its potential importance for ecosystem functionality.

Fig. 1Murphy et al. (2016) demonstrated that exposing Pseudomonas aeroginosa ( $n=3$ plates) and Staphylococcus aureus $(n=3)$ to frequencies of $0.1,0.8$, and $1.6 \mathrm{kHz}$ for $48 \mathrm{~h}$ resulted in a significant increase in biofilm formation (compared to the control). Greatest growth for $P$. aeruginosa was recorded at $0.8 \mathrm{kHz}$, and for $S$. aureus it was $1.6 \mathrm{kHz}$. This study did not use decibel units in their assessment but the inter-species variation in growth rate was dependent on sound frequency. Again, the sample sizes are low, as such, the results should be interpreted with caution. Inter-species variation in growth was also shown in a study by Sarvaiya and Kothari (2015). The authors exposed Chromobacterium violaceum, Serratia marcescens, Staphylococcus aureus, and Streptococcus pyogenes to music at a frequency range of 38-689 Hz. All bacteria increased in growth (3-40\% higher) except for $S$. marcescens, which decreased in growth $(-8 \%)$ and pigment (prodigiosin) production (-16\%). C. violaceum's growth increased by $40 \%$ and prodigiosin pigment production increased by $66 \%$. The authors suggest that observed alteration in pigment production is not entirely due to growth, but more likely quorum 


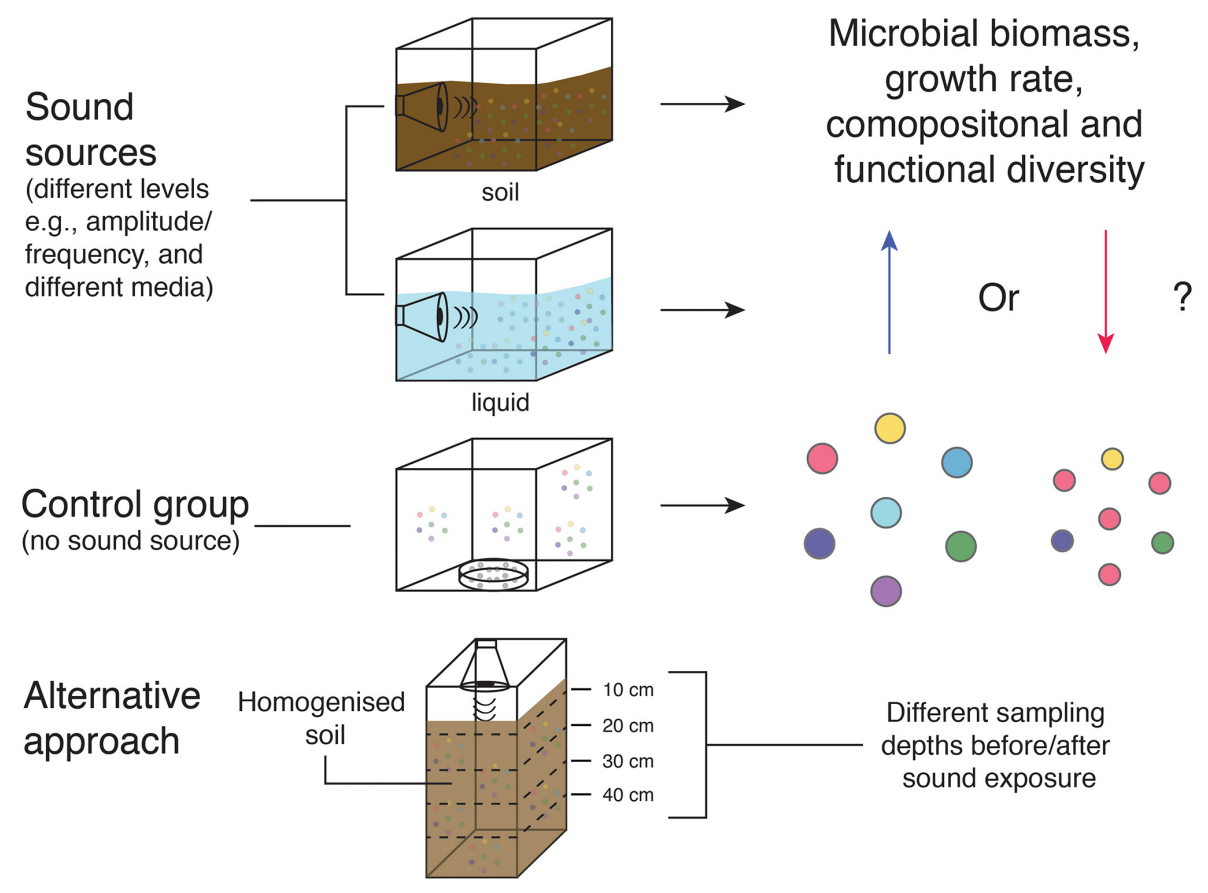

FIGURE 1 | Schematic of sound exposure experiments. Gu et al. (2016) investigated biomass and growth rate of a single taxa. However, similar experiments could be carried out to investigate potential changes in microbial community compositional and functional diversity, whilst testing different interfaces/media that may have an important effect on sound propagation.

sensing (i.e., intercellular communication) affected by sound. These studies imply that anthropogenic sound exposure can affect microbial growth, biomass and synthesis of intracellular molecules via a range of pathways, and that certain frequencies and amplitudes may favor some microbial species over others.

A recent mouse-model study demonstrated adverse effects of noise pollution on the gut microbiome (Cui et al., 2020). They used 16S rRNA sequencing to characterize the gut microbiome and the Tax4Fun package in $\mathrm{R}$ to predict metagenome content. The authors found that in mice $(n=40)$ exposed to white noise at $98 \mathrm{~dB}$ (frequency of $20 \mathrm{kHz}$ for $4 \mathrm{~h}$ per day, for 30 days) but not the control groups, bacterial-encoded functional profiles included an increase in phospholipid and galactose metabolism, oxidative stress, and cell senescence which corresponded with systemic inflammation. The authors suggest this may have implications for early onset Alzheimer's disease. This study shows interesting results that could have important implications for public health. In subsequent studies, greater value could be added by using whole genome sequencing instead of amplicon-functional profiling approach, and focusing on relationship directionality.

Another study investigated glucose metabolism and gut microbiota-host inflammatory homeostasis in rats $(n=64)$ (Cui et al., 2016). The authors found that chronic noise (100 dB, 400 $\mathrm{Hz}-6.3 \mathrm{kHz}, 4 \mathrm{~h}$ a day for 30 days) altered the percentage of Proteobacteria and Actinobacteria in the gut and corresponding abnormalities in glucose and insulin regulation relative to controls. They suggest that anthropogenic noise exposure could have cumulative effects on diabetes onset due to microbiome compositional changes and intestinal inflammation. Once again, these results could have important implications for public health by improving our understanding of the factors that may contribute to diabetes. It is worth mentioning that although appropriate in 2016 (time of the study), the approach used to characterize the microbiome--via operational taxonomic units with $97 \%$ similarity--is now considered to be outdated. Exact Sequence Variants (ESV) may provide a richer taxonomic picture (Callahan et al., 2017), and whole genome sequencing, although more expensive, would enhance functional profiling.

\section{Algae, Fungi, and Zooplankton}

We found 2 studies pertaining to the exposure of sound on algae, 2 for fungi (1 paper was excluded due to a lack of data and relevance), and 1 for zooplankton. Cai et al. (2016) exposed the microalga Picochlorum oklahomensis to anthropogenic sound at $90 \mathrm{~dB}$ and $1.1,2.2$, and $3.3 \mathrm{kHz}$ for $3 \mathrm{~h}$ a day for 30 days. The authors found that all frequencies increased biomass compared to the control, but that $2.2 \mathrm{kHz}$ was the most effective (e.g., oil yield of $40.37 \mathrm{~g} / \mathrm{L}$ compared to the control of $31.66 \mathrm{~g} / \mathrm{L}$ ). The sample size is not clear, although it appears to be low at only 2 replicates per treatment. As the authors state, an expansion of the study is needed to decipher the mechanism responsible for the increased biomass due to the complexity of interacting variables. Given that lipid accumulation is a stress response to nitrogen limitation, measurements of nutrient uptake would be an interesting complement to growth data. The results of this study align with previous reports by Jiang et al. (2012) who cultured Chlorella pyrenoidosa for 7 days 
with sound exposure at $80 \mathrm{~dB}$ and frequencies of $0.2,0.4,0.7$, 1,2 and $6 \mathrm{kHz}$. They found that C. pyrenoidosa growth due to sound exposure was $30 \%$ higher than the control, with an optimal frequency between 0.4 and $1 \mathrm{kHz}$. Again, it is not clear what the sample size was for this study, therefore, the results should be interpreted with caution. Kumar (2020) found that the biomass of the yeast Saccharomyces cerevisiae increased significantly following sound exposure of $0.1-10 \mathrm{kHz}$ for $8 \mathrm{~h}$ compared to a control. Once again, the sample size is not clearly stated and the study is difficult to interpret. A challenge is that many of these studies rely on optical density (OD) to measure microbial growth. OD measurements are assumed to be proportional to sample concentration (cell numbers) (Stevenson et al., 2016). Taken in tandem with cell counts and dry cell weight, the impact on cell growth could be interpreted with more certainty. Interestingly, Aggio et al. (2012) used metabolomics to compare the physiology of yeast cells $(n=15)$ exposed to high $(10 \mathrm{kHz})$ and low $(0.1 \mathrm{kHz})$ frequency sonic vibration at $90 \mathrm{~dB}$. All stimuli increased the growth rate of the yeast by $12 \%$ but reduced biomass production by $14 \%$, and different frequencies induced different metabolomic responses. Other studies have shown that sound can evoke physiological reactions in plants (e.g., via gene expression in Arabidopsis) (Jung et al., 2018) and potentially enhance growth (Hassanien et al., 2014). Future studies could explore this from a "holobiont" (collective host and symbiotic organisms) perspective and investigate the directionality of the relationship (e.g., microbe -> host and/or host $->$ microbe?).

Finally, it is worth noting that anthropogenic noise pollution (e.g., from seismic operations) has been shown to adversely affect zooplankton. McCauley et al. (2017) demonstrated that following seismic air gun exposure, there were 2-3 times more dead zooplankton $(n=78)$ for all taxa compared with controls, and up to $1.2 \mathrm{~km}$ away from the source. All krill larvae found in the exposed samples were dead. This suggests potentially underacknowledged implications for ocean ecosystem functionality and productivity and warrants further research.

\section{THE EFFECTS OF ARTIFICIAL LIGHT POLLUTION ON MICROORGANISMS}

Artificial light pollution can also have important ecological and public health impacts. We found 8 papers pertaining to the effects of artificial light on microorganisms.

\section{Biofilms and Sediments}

Maggi et al. (2020a) explored the effects of ALAN (using LED lamps with a mean of 27 lux to mimic coastal urban lighting) on marine biofilms (microphytobenthos). They observed biofilm quadrats $(n=24)$ over a period of 204 days. They showed a significant increase in temporal variance of maximum photosynthetic efficiency under ALAN. This suggests that ALAN may differentially affect certain groups in microbial biofilms due to species-specific sensitivities. The authors conclude that future studies should aim to understand the interactions between ALAN and other anthropogenic disturbances on microbiomes. Hölker et al. (2015) investigated the response of microbial communities in freshwater sediments to artificial light exposure $(n=30)$. They used $70 \mathrm{~W}$ high-pressure sodium lamps $\left(2,000 \mathrm{~K}, 96 \mathrm{~lm} \mathrm{~W}^{-1}\right)$ and nocturnal light levels ranged from 13.3 to 16.5 lux at the water surface and 6.8-8.5 lux at the sediment surface $(50 \mathrm{~cm}$ depth). Over a 1 year period they observed an increased abundance of phototrophic taxa (diatoms, Cyanobacteria and green algae) in sediments after 5 months of ALAN compared to the control. The authors suggest that ALAN over waterbodies could reduce diurnal fluctuations and has the potential to transform freshwater systems to nocturnal carbon sinks. Further studies are needed to ascertain the full ecological impacts (both direct and indirect) of this process.

\section{Coral Microbiome}

Baquiran et al. (2020) investigated the effects of ALAN on the coral Acropora digitifera and its microbiome. The authors exposed corals $(n=45)$ to ALAN (27-45 lux) for 2 months. They found that microbial diversity remained stable after ALAN exposure, but certain taxa in the families Rhodobacteraceae, Caulobacteraceae, Burkholderiaceae, Lachnospiriaceae, and Ruminococcaceae significantly increased in exposed corals. The observed compositional stability of the coral microbiome in this study may indicate physiological plasticity of different microbes, potentially allowing the community to buffer environmental disturbance with continued provision of important metabolites. Further studies should investigate how longer-term ALAN exposure affects the corals and whether the observed changes in microbial families has positive or negative outcomes for coral ecosystems. Additional research on the potential impacts of ALAN-induced microbiome changes on gene expression of corals would also be beneficial. Rosenberg et al. (2019) found that corals exposed to ALAN have 25 times more differentially expressed genes that regulate cell cycle, proliferation, growth and protein synthesis that may act as a chronic disturbance.

\section{The Gut Microbiome}

A recent mouse-model study $(n=28)$ demonstrated that prolonged artificial light exposure can significantly alter the gut microbiome and promote non-alcoholic fatty liver disease (NAFLD) (Wei et al., 2020). The authors used white fluorescent light tubes with a wavelength of $400 \sim 560 \mathrm{~nm}$ set at 200 lux. They compared normal light-dark ratios with constant light exposure and found that constant light significantly altered gut microbiome composition and promoted functional pathways related to type-2 diabetes in addition to promoting obesity and NAFLD. Future studies would likely benefit from whole genome sequencing as opposed to OTU analysis. However, this study points to important public health implications of artificial light exposure.

Artificial light has also been shown to alter gut microbiome composition in the Eurasian tree sparrow Passer montanus $(n=40)$ (Jiang et al., 2020). In this study light (400-1,400 lux for $12 \mathrm{~h}$, followed by 8 lux for $12 \mathrm{~h}$ ) reduced bacterial alpha diversity (Shannon 5.70) and significantly affected melatonin synthesis compared to the dark control (Shannon 6.96). As light pollution affects melatonin, which itself helps to regulate 
the hypothalamus-pituitary-adrenal and microbiota-gut-brain axes (Anderson and Maes, 2015), this could potentially have important cascading impacts on physiological and psychological health. There is a clear deficit in studies exploring the effects of light pollution on the human microbiome, and as such, more research in this area is warranted.

\section{DISCUSSION}

This mini-review shows that a limited number of studies have been carried out to investigate the effects of anthropogenic sound and artificial light pollution on microbiomes. However, the studies do indicate that anthropogenic sound and artificial light may have important influences on microbially mediated ecosystem processes and human health. Both forms of pollution are considered to be global health issues and can affect ecosystem composition and functionality. Considerably more research is needed to gain a better understanding of the effects of sound and light pollution on microbiomes. Indeed, ecosystems are under immense pressure from various forms of degradation. By understanding the effects and processes involved, we can start to design appropriate mitigation strategies. Contra to this, we could potentially utilize any positive sound/light-induced microbial effects to improve ecosystem stability and human health outcomes. The studies mentioned in this paper lay the foundations for important future work in microbial ecology and public health.

Understanding that different sound exposures (e.g., amplitude, frequency, durations) induce inter-species variation in growth, biomass and synthesis of intracellular molecules could have important implications for many ecological processes across trophic levels. We also do not yet fully understand the mechanisms by which sound stimulates microbial growth, as suggested by $\mathrm{Gu}$ et al. (2016). Mechanosensitive channels on bacterial cell membranes might be involved in signal transduction, but gaining a better understanding will enable optimization of the processes or mitigation for adverse exposures. The indication that increased bacterial resistance to ampicillin was attributed to low frequency anthropogenic noise certainly warrants further research due to its potential importance in the fight against antibiotic resistance. Understanding how sound affects plant-microbe (or animal-microbe) interactions as indicated by Kim (2016), could be extremely important given that both anthropogenic sound pollution and ecosystem degradation are increasing globally (Figure 2). Plant health is imperative and microbial interactions are essential to the provision of multiple ecosystem services (Guerra et al., 2020). An interesting line of enquiry could be to investigate whether sound pollution influences environmental microbiome assembly and intercellular signaling to the point where it affects plant health and (bioacoustic) communication. The effects of anthropogenic sound on human and non-human animal microbiomes also merits a deeper investigation. Exposure to biodiverse natural environments alters the human microbiome with potential benefits to human health (Roslund et al., 2020; Selway et al., 2020). Exposure routes may differ depending on ecological characteristics such as vegetation complexity and height (Robinson et al., 2020). Another interesting line of enquiry is whether different levels of urban sound pollution affect the composition, assembly and exposure routes of microorganisms.

ALAN is also likely to affect human health and ecosystem functionality via impacts on the microbiome. Although initial work suggests that ALAN significantly affects marine and freshwater bodies, it is unclear whether the impacts are negative in the long-term. Indeed, Hölker et al. (2015) suggests ALAN has the potential to transform freshwater systems to nocturnal carbon sinks. Further studies to ascertain the multidimensional ecological impacts of ALAN are needed, because it could potentially have important unforeseen multi-trophic impacts. Indeed, it is a similar story for corals because the studies report variable results. However, as coral reefs are under immense pressure, this is an important area of research.

This mini-review highlights that additional research is needed to unravel the effects of light pollution on the human microbiome. Indications from the studies suggest that artificial light could adversely impact physiological processes via the microbiome, and potentially contribute toward metabolic diseases. If anthropogenic sound and ALAN affect humanenvironmental microbiome exposure and influence human physiology directly, there could also be important social equity issues to investigate. Social disparities in exposure to anthropogenic sound pollution have been documented

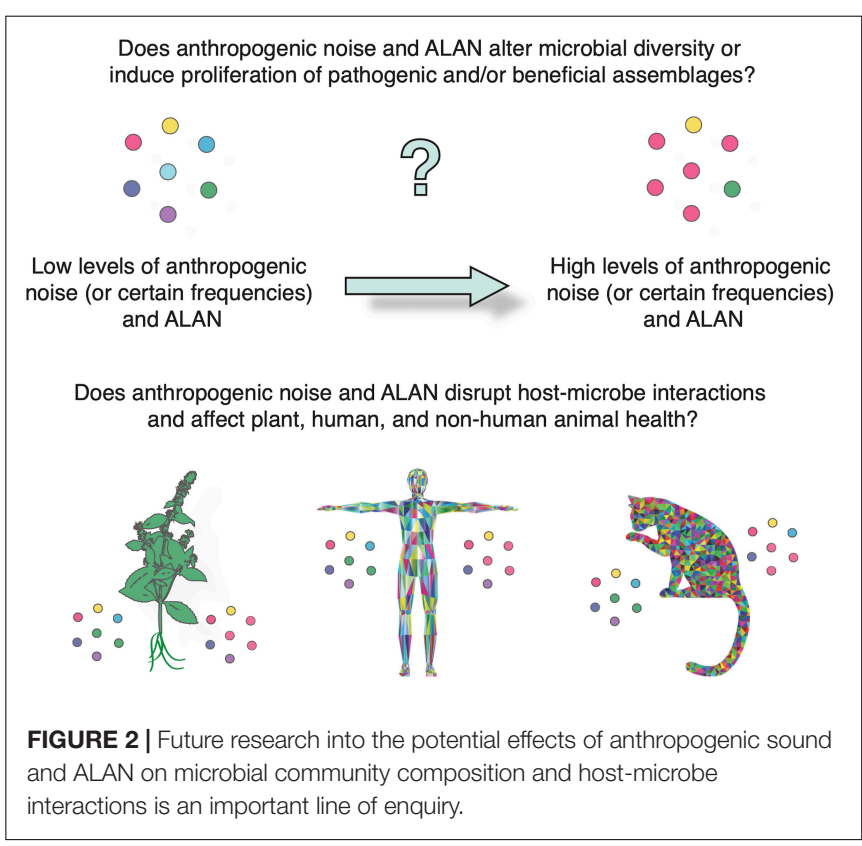

BOX 1| The photo-sonic restoration hypothesis.

If anthropogenic sound and light disrupt microbiome assembly, potentially favoring certain adaptable species and reducing functional diversity, this could have important ecosystem and health implications. Therefore, does restoring natural levels of light and sound help to restore microbiomes and ecosystem stability? We hereby propose the photo-sonic restoration hypothesis. 
(Dreger et al., 2019). Therefore, in some cases, the impacts of exposure will also be unequally distributed across different social groups. This warrants further research.

Many of the studies in this mini-review suffered from modest sample sizes, suboptimal experimental designs (e.g., lack of negative controls, cell counts and particle sizing), and some of the bioinformatics approaches used are now outdated. These factors should be improved in future studies. However, it is clear that anthropogenic sound pollution and ALAN have the potential to influence ecosystems and human health via interactions with microbiomes. This is an emerging and severely underexplored field of research that could have important implications for global ecosystems and public health. There is also an intriguing hypothesis to consider (Box 1).

\section{REFERENCES}

Aggio, R. B. M., Obolonkin, V., and Villas-Bôas, S. G. (2012). Sonic vibration affects the metabolism of yeast cells growing in liquid culture: a metabolomic study. Metabolomics 8, 670-678. doi: 10.1007/s11306-011-0360-x

Anderson, G., and Maes, M. (2015). The gut-brain axis: the role of melatonin in linking psychiatric, inflammatory and neurodegenerative conditions. $A d v$. Integ. Med. 2, 31-37. doi: 10.1016/j.aimed.2014.12.007

Baquiran, J. I. P., Nada, M. A. L., Campos, C. L. D., Sayco, S. L. G., Cabaitan, P. C., Rosenberg, Y., et al. (2020). The prokaryotic microbiome of Acropora digitifera is stable under short-term artificial light pollution. Microorganisms 8:1566.

Cai, W., Dunford, N. T., Wang, N., Zhu, S., and He, H. (2016). Audible sound treatment of the microalgae Picochlorum oklahomensis for enhancing biomass productivity. Bioresour. Technol. 202, 226-230. doi: 10.1016/j.biortech.2015.12. 019

Callahan, B. J., McMurdie, P. J., and Holmes, S. P. (2017). Exact sequence variants should replace operational taxonomic units in marker-gene data analysis. ISME J. 11, 2639-2643. doi: 10.1038/ismej.2017.119

Cavicchioli, R., Ripple, W. J., Timmis, K. N., Azam, F., Bakken, L. R., Baylis, M., et al. (2019). Scientists' warning to humanity: microorganisms and climate change. Nat. Rev. Microbiol. 17, 569-586.

Chepesiuk, R. (2005). Decibel hell: the effects of living in a noisy world. Environ. Health Perspect. 113, 34-41.

Cryan, J. F., O’Riordan, K. J., Cowan, C. S., Sandhu, K. V., Bastiaanssen, T. F., Boehme, M., et al. (2019). The microbiota-gut-brain axis. Physiol. Rev. 99, 1877-2013.

Cui, B., Chi, H., Cao, W., Su, D., Yang, H., Li, Z., et al. (2020). Noise ExposureInduced Intestinal Flora Dysbiosis Disrupts Homeostasis of oxi-Inflamm-Barrier in the Gut-Brain Axis of APP/PS1 Mice: Implications for Early Onset Alzheimer's Disease. Available online at: https://doi.org/10.21203/rs.3.rs-19045/v1 (accessed January 5, 2021).

Cui, B., Gai, Z., She, X., Wang, R., and Xi, Z. (2016). Effects of chronic noise on glucose metabolism and gut microbiota-host inflammatory homeostasis in rats. Sci. Rep. 6:36693.

Díaz, J., López-Bueno, J. A., López-Ossorio, J. J., Gónzález, J. L., Sánchez, F., and Linares, C. (2020). Short-term effects of traffic noise on suicides and emergency hospital admissions due to anxiety and depression in Madrid (Spain). Sci. Total Environ. 710:136315. doi: 10.1016/j.scitotenv.2019.136315

Dreger, S., Schüle, S. A., Hilz, L. K., and Bolte, G. (2019). Social inequalities in environmental noise exposure: a review of evidence in the WHO European region. Int. J. Environ. Res. Public Health 16:1011. doi: 10.3390/ijerph1606 1011

Falchi, F., Furgoni, R., Gallaway, T. A., Rybnikova, N. A., Portnov, B. A., Baugh, K., et al. (2019). Light pollution in USA and Europe: the good, the bad and the ugly. J. Environ. Manag. 248:109227. doi: 10.1016/j.jenvman.2019. 06.128

Gendron, G., Tremblay, R., Jolivet, A., Olivier, F., Chauvaud, L., Winkler, G., et al. (2020). Anthropogenic boat noise reduces feeding success in winter flounder

\section{AUTHOR CONTRIBUTIONS}

JR contributed to the conception, design of the article, wrote the first draft of the manuscript, and produced the visualizations and figures. JR, RC, and BP contributed to the critical review and manuscript revisions. All authors read and approved the submitted version.

\section{FUNDING}

JR was undertaking a Ph.D. through the White Rose Doctoral Training Partnership (WRDTP), funded by the Economic and Social Research Council (ESRC).

larvae (Pseudopleuronectes americanus). Environ. Biol. Fishes 103, 1079-1090. doi: 10.1007/s10641-020-01005-3

Gu, S., Zhang, Y., and Wu, Y. (2016). Effects of sound exposure on the growth and intracellular macromolecular synthesis of E. coli k-12. PeerJ 4:e1920. doi: $10.7717 /$ peerj. 1920

Guerra, C. A., Heintz-Buschart, A., Sikorski, J., Chatzinotas, A., Guerrero-Ramírez, N., Cesarz, S., et al. (2020). Blind spots in global soil biodiversity and ecosystem function research. Nat. Commun. 11:3870.

Hassanien, R. H., Hou, T. Z., Li, Y. F., and Li, B. M. (2014). Advances in effects of sound waves on plants. J. Integ. Agric. 13, 335-348. doi: 10.1016/s20953119(13)60492-x

Hatori, M., Gronfier, C., Van Gelder, R. N., Bernstein, P. S., Carreras, J., Panda, S., et al. (2017). Global rise of potential health hazards caused by blue light-induced circadian disruption in modern aging societies. NPJ Aging Mech. Dis. 3:9.

Hölker, F., Wurzbacher, C., Weißenborn, C., Monaghan, M. T., Holzhauer, S. I., and Premke, K. (2015). Microbial diversity and community respiration in freshwater sediments influenced by artificial light at night. Philos. Trans. R. Soc. Lond. B Biol. Sci. 370:20140130. doi: 10.1098/rstb.2014.0130

Janson, E., Johannessen, A., Holm, M., Franklin, K., Holst, G. J., Gislason, T., et al. (2020). Insomnia associated with traffic noise and proximity to traffic-a crosssectional study of the respiratory Health in Northern Europe III population. J. Clin. Sleep Med. 16, 545-552. doi: 10.5664/jcsm.8274

Jiang, J., He, Y., Kou, H., Ju, Z., Gao, X., and Zhao, H. (2020). The effects of artificial light at night on Eurasian tree sparrow (Passer montanus): behavioral rhythm disruption, melatonin suppression and intestinal microbiota alterations. Ecol. Indic. 108:105702. doi: 10.1016/j.ecolind.2019.105702

Jiang, S., Rao, H., Chen, Z., Liang, M., and Li, L. (2012). Effects of sonic waves at different frequencies on propagation of Chlorella pyrenoidosa. Agric. Sci. Technol. 13, 2197-2201.

Jung, J., Kim, S. K., Kim, J. Y., Jeong, M. J., and Ryu, C. M. (2018). Beyond chemical triggers: evidence for sound-evoked physiological reactions in plants. Front. Plant Sci. 9:25.

Kim, H. W. (2016). The effects of low frequency noise on the growth and resistance to antibiotics of soil bacteria and E. coli. APEC Youth Scientist J. 8, 1-10. doi: 10.1007/978-3-319-23534-9_1

Kowalski, K., and Mulak, A. (2019). Brain-gut-microbiota axis in Alzheimer's disease. J. Neurogastroenterol. Motil. 25, 48-60.

Kumar, A., Kumar, P., Mishra, R. K., and Shukla, A. (2018). "Study of air and noise pollution in mega cities of India," in Environmental Pollution, eds V. P. Singh, S. Yadav, and R. N. Yadava (Berlin: Springer), Available online at: springer.com/gp/book/9789811057915.

Kumar, R. (2020). Impact of regulative noise exposure to biodiesel production due to enhanced lipid droplet production in Saccharomyces cerevisiae: preliminary results from a laboratory experiment. bioRxiv. [preprint] doi: 10.1101/2020.11. 22.387878

Li, M., Fang, A., Yu, X., Zhang, K., He, Z., Wang, C., et al. (2020). Microbiallydriven sulfur cycling microbial communities in different mangrove sediments. Chemosphere 13:128597. doi: 10.1016/j.chemosphere.2020.128597 
Luo, J., Siemers, B. M., and Koselj, K. (2015). How anthropogenic noise affects foraging. Glob. Chang. Biol. 21, 3278-3289. doi: 10.1111/gcb.12997

Maggi, E., Bertocci, I., and Benedetti-Cecchi, L. (2020a). Light pollution enhances temporal variability of photosynthetic activity in mature and developing biofilm. Hydrobiologia 847, 1793-1802. doi: 10.1007/s10750-019-04102-2

Maggi, E., Bongiorni, L., Fontanini, D., Capocchi, A., Dal Bello, M., Giacomelli, A., et al. (2020b). Artificial light at night erases positive interactions across trophic levels. Funct. Ecol. 34, 694-706. doi: 10.1111/1365-2435.13485

Mason, J. T., McClure, C. J., and Barber, J. R. (2016). Anthropogenic noise impairs owl hunting behavior. Biol. Conserv. 199, 29-32. doi: 10.1016/j.biocon.2016.04. 009

McCauley, R. D., Day, R. D., Swadling, K. M., Fitzgibbon, Q. P., Watson, R. A., and Semmens, J. M. (2017). Widely used marine seismic survey air gun operations negatively impact zooplankton. Nat. Ecol. Evol. 1:0195.

Min, J. Y., and Min, K. B. (2018). Outdoor light at night and the prevalence of depressive symptoms and suicidal behaviors: a cross-sectional study in a nationally representative sample of Korean adults. J. Affect. Disord. 227, 199-205. doi: 10.1016/j.jad.2017.10.039

Münzel, T., Schmidt, F. P., Steven, S., Herzog, J., Daiber, A., and Sørensen, M. (2018). Environmental noise and the cardiovascular system. J. Am. Coll. Cardiol. 71, 688-697.

Murphy, M. F., Edwards, T., Hobbs, G., Shepherd, J., and Bezombes, F. (2016). Acoustic vibration can enhance bacterial biofilm formation. J. Biosci. Bioeng. 122, 765-770. doi: 10.1016/j.jbiosc.2016.05.010

Owens, A. C., Cochard, P., Durrant, J., Farnworth, B., Perkin, E. K., and Seymoure, B. (2020). Light pollution is a driver of insect declines. Biol. Conserv. 241:108259. doi: 10.1016/j.biocon.2019.108259

Prescott, S. L., Larcombe, D. L., Logan, A. C., West, C., Burks, W., Caraballo, L., et al. (2017). The skin microbiome: impact of modern environments on skin ecology, barrier integrity, and systemic immune programming. World Allergy Organ. J. 10:29. doi: 10.1186/s40413-017-0160-5

Renz, H., and Skevaki, C. (2020). Early life microbial exposures and allergy risks: opportunities for prevention. Nat. Rev. Immunol. 21, 177-191. doi: 10.1038/ s41577-020-00420-y

Robinson, J. M., and Jorgensen, A. (2020). Rekindling old friendships in new landscapes: the environment-microbiome-health axis in the realms of landscape research. People Nat. 2, 339-349. doi: 10.1002/pan3.10082

Robinson, J. M., Cando-Dumancela, C., Liddicoat, C., Weinstein, P., Cameron, R., and Breed, M. F. (2020). Vertical stratification in urban green space aerobiomes. Environ. Health Perspect. 128:117008. doi: 10.1289/ehp7807

Rook, G. A. (2013). Regulation of the immune system by biodiversity from the natural environment: an ecosystem service essential to health. Proc. Nat. Acad. Sci. 110, 18360-18367. doi: 10.1073/pnas.1313731110

Rosenberg, Y., Doniger, T., and Levy, O. (2019). Sustainability of coral reefs are affected by ecological light pollution in the Gulf of Aqaba/Eilat. Commun. Biol. $2: 289$.

Roslund, M. I., Puhakka, R., Grönroos, M., Nurminen, N., Oikarinen, S., Gazali, A. M., et al. (2020). Biodiversity intervention enhances immune regulation and health-associated commensal microbiota among daycare children. Sci. Adv. 6:eaba2578. doi: 10.1126/sciadv.aba2578

Sarvaiya, N., and Kothari, V. (2015). Effect of audible sound in form of music on microbial growth and production of certain important metabolites. Microbiology 84, 227-235. doi: 10.1134/s0026261715020125

Selway, C. A., Mills, J. G., Weinstein, P., Skelly, C., Yadav, S., Lowe, A., et al. (2020). Transfer of environmental microbes to the skin and respiratory tract of humans after urban green space exposure. Environ. Int. 145:106084. doi: 10.1016/j.envint.2020.106084

Shaobin, G., Wu, Y., Li, K., Li, S., Ma, S., Wang, Q., et al. (2010). A pilot study of the effect of audible sound on the growth of Escherichia coli. Colloids Surf. B Biointerfaces 78, 367-371. doi: 10.1016/j.colsurfb.2010.02.028

Sharma, A., and Im, S. H. (2020). Special issue on the human microbiome: from symbiosis to therapy. Exp. Mol. Med. 52, 1361-1363. doi: 10.1038/s12276-02000488-5

Song, S., Chang, Y., Wang, D., Jiang, T., Feng, J., and Lin, A. (2020). Chronic traffic noise increases food intake and alters gene expression associated with metabolism and disease in bats. J. Appl. Ecol. 57, 1915-1925. doi: 10.1111/13652664.13710

Stevenson, K., McVey, A. F., Clark, I. B. N., Swain, P. S., and Pilizota, T. (2016). General calibration of microbial growth in microplate readers. Sci. Rep. 6:38828.

Tabraiz, S., Ahmad, S., Shehzadi, I., and Asif, M. B. (2015). Study of physiopsychological effects on traffic wardens due to traffic noise pollution; exposureeffect relation. J. Environ. Health Sci. Eng. 13:30.

Trivedi, P., Leach, J. E., Tringe, S. G., Sa, T., and Singh, B. K. (2020). Plantmicrobiome interactions: from community assembly to plant health. Nat. Rev. Microbiol. 18, 607-621. doi: 10.1038/s41579-020-0412-1

Vitkauskaite, G., and Grubliauskas, R. (2018). Perforated sound-absorbing constructions acoustic performance test and noise modeling. Energy Procedia 147, 288-294. doi: 10.1016/j.egypro.2018.07.094

Wale, M. A., Simpson, S. D., and Radford, A. N. (2013). Noise negatively affects foraging and antipredator behaviour in shore crabs. Anim. Behav. 86, 111-118. doi: 10.1016/j.anbehav.2013.05.001

Wei, L., Yue, F., Xing, L., Wu, S., Shi, Y., Li, J., et al. (2020). Constant light exposure alters gut microbiota and promotes the progression of steatohepatitis in high fat diet rats. Front. Microbiol. 11:1975.

Conflict of Interest: The authors declare that the research was conducted in the absence of any commercial or financial relationships that could be construed as a potential conflict of interest.

Copyright (C) 2021 Robinson, Cameron and Parker. This is an open-access article distributed under the terms of the Creative Commons Attribution License (CC BY). The use, distribution or reproduction in other forums is permitted, provided the original author(s) and the copyright owner(s) are credited and that the original publication in this journal is cited, in accordance with accepted academic practice. No use, distribution or reproduction is permitted which does not comply with these terms. 